\title{
A reliability approach for the MEA power system architecture design optimization problem
}

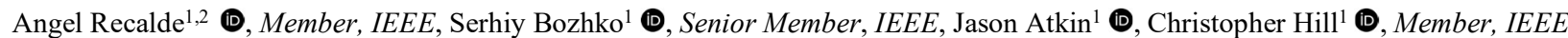 \\ ${ }^{1}$ School of Electrical and Electronic Engineering \\ The University of Nottingham \\ Nottingham, United Kingdom \\ \{angel.recalde, jason.atkin, serhiy.bozhko, c.hill\}@nottingham.ac.uk \\ ${ }^{2}$ Faculty of Electrical Engineering and Computer Science, FIEC, GISE Group \\ Escuela Superior Politécnica del Litoral, ESPOL \\ Campus Gustavo Galindo Km 30.5 Via Perimetral, P.O. Box 09-01-5863, Guayaquil, Ecuador \\ arecalde@espol.edu.ec
}

\begin{abstract}
In the literature, there has been thorough research on reliability-based design in different fields. However, aircraft power system investigations have mostly dealt with sub-system optimization. Hence, there is considerable potential to excel innovative formulations that could promote rapid adoption of new technologies, thus favouring continuous novel solution exploration. This paper reviews reliability-based architecture design optimization concepts which have been utilised in alternate applications and could jointly be applied to this MEA problem. Furthermore, it proposes a simplified mathematical formulation to illustrate the ideas behind such approach.
\end{abstract}

Keywords-more electric aircraft, network design optimization, reliability, aircraft power system architecture

\section{INTRODUCTION}

The More Electric Aircraft (MEA) is a concept that defines the future of aircraft electrification [1]. Drivers such as greener environments [2], fuel consumption reduction [3], and potential efficiency improvement have been extensively translated into high-level innovative research across all fields within aerospace sciences. Current examples are additive manufacturing and composite engineering which have led the path to improve material performance and airplane aerodynamic characteristics [4]. However, the aircraft electric power network plays a major role and could substantially contribute to achieving global ambitious environmental goals due to its importance in converting and supplying energy on-board [5]. Moreover, there are a diverse variety of novel electro-mechanical options that could potentially replace aircraft hydraulic and pneumatic systems [6]. But this innovation involves an appropriate power system design in order to be attractive for business adoption. Indeed, the aerospace industry demands a rigorous compliance with safety requirements, while being restricted by highlyconstrained techno-economic constraints [1].

An aircraft power network is a complex system that consists of thousands of interconnected components, which can be roughly classified into loads, onboard and ground power sources, emergency network, energy storage devices, control hardware and software, instrumentation networks, and protection devices [7]. Its design deserves a huge deployment of resources and expertise which is not always directly related to electrical and electronic engineering. Hence, for the adoption of new technologies in MEA, it is compulsory to conceive, at the early design stage, an abstract characterization of requirements that could be drafted into a mathematical optimizable formulation. Firstly, the definition of building blocks [8] of attributes provides a simplified representation of components [9]. Secondly, it is possible to link together both performance features and network connectivity rules to devise a system prototype with desired characteristics. The quality of the proposal could be evaluated in terms of expandability, adaptability [10], and reliability Now, it is desirable that this design route could come to a feasible optimum solution if it were established as an optimization problem. Although emerging technologies often encounter adoption barriers, data techniques such as accelerated life testing analysis could help in supporting rapid acceptance. Due to the fact that current technology is rapidly evolving, it is critical to guarantee system reliability at all times. As the MEA concept represents a change of paradigm in aerospace, safety and reliability arguments will definitely drive approval and advancements. Thus, it is necessary to incorporate reliability concepts in the aircraft power system architecture design problem. This paper aims to provide a simplified review of reliability-based architecture design optimization concepts that could be employed in the MEA power system design problem. This paper is organized as follows. Section II details reliability optimization concepts and types; section III reviews design approaches and reliability characterization encountered in some engineering fields in the literature. In section IV, a MEA reliability-oriented power system design optimization problem is proposed. Finally, section V summarizes the conclusions.

\section{RELIABILITY OPTIMIZATION CONCEPTS AND TYPES}

Reliability-based architecture design optimization is a complex process that involves not only the formulation of an appropriate objective, but also the establishment of techniceconomic requirements that could be introduced algebraically as mathematical constraints [11]. Generally, reliability comprises the study of the overall performance of a system containing failure-prone elements. In the MEA context, reliability is the aircraft's ability to perform a complete flight mission. It is usually defined with a measurable performance index. 
Measurements of system performance could be broadly divided into four types: reliability indices, availability, meantime-between-failures MTBF, and percentile life $\tau_{\text {life }}$ [12]. Reliability index $R_{S}$ is a metric that measures the ability of the system to perform its required function under given conditions for a specified time. On the other hand, availability is the proportion of time a system is in an operating condition, and it is mostly used for maintained systems. MTBF is the mean life of a system and is commonly used as an index to compare several system performances, although it does not provide as much information as the system's reliability $R_{S}$. Finally, $\tau_{\text {life }}$ is the mission time at which the system reliability meets a specific value [13]. Thus, there is a functional relation between reliability and $\tau_{\text {life }}$. However, $\tau_{\text {life }}$ is preferable when the system mission time is indeterminate.

While these metrics are commonly defined on a failuresuccess operation of the system, there is the possibility to include other types of operation that are not necessarily success or failure. An example could be a stand-by operation. These operational circumstances are known as states. Then, a $b i$-state system could be either failed or OK (success); on the other hand, a system could have multiple-states, including failed or OK naturally. Despite that, system failure not only relies on its own characteristics, but also on external influence. Considering that, some authors have utilized survivability as a concept to describe the ability of a multi-state system to tolerate both internal failures and external attacks [12]. Nevertheless, a $b i$ state definition has been extensively used in reliability engineering for system performance assessment.

Considering reliability index $R_{S}$ as the main metric to characterize the system performance, optimization can be performed in several ways. Hence, power system reliability optimization problems could be broadly classified into four types, as shown in Table I and explained in Section II-A. The main idea behind these formulations is to optimize the system performance including technical and economic constraints.

\section{A. Formulation types in optimal reliability design}

Considering the reliability index $R_{S}$ as the main performance metric to optimize, four types of optimization formulations can be stated. These formulations are shown in Table I and are described below.

1) Type 1: This formulates a single-objective reliability optimization problem with $R_{S}$ as the system reliability objective function to be optimized directly. The technical and economic constraints are defined in the form $g_{i}(x) \leq b_{i}$, where $g_{i}(x)$ is an expression that represent the amount of technical or economic resource utilized (there are $m$ number of resource contraints, so that $i \in\{1 . . m\}$ ), and $b_{i}$ represent the constrained target. Thus, each resource is constrained to be no worse than some threshold value. For instance, weight is a resource that could be introduced as a constraint in this type of formulation in the form $g_{i}(x) \leq b_{i}$.

2) Type II: This type also formulates a single-objective reliability optimization problem, but it optimizes a selected technical or economic resource directly instead of the system's reliability. Henceforth, the selected resource will be referred as the resource cost $C_{S}$, and it is optimized while ensuring that reliability $R_{S}$ meets a specified threshold $R_{0}$, via the addition of a constraint $R_{S} \geq R_{0}$, known as hard-constraint [12]. Here, $C_{S}$ is not only related to monetary expenses (operation \& maintenance), but also to other resources, e.g. physical design constraints. For example, weight could be used as the resource to minimize, such as in a system to minimize weight while ensuring that reliability and other cost requirements are met. Similar to type 1 , other resource costs are usually constrained by a less-or-equal-than function constraint $g_{i}(\mathrm{x}) \leq b_{i}$, where $b_{i}$ represents a constrained resource target.

\begin{tabular}{|c|c|}
\hline Type & Formulations with Reliability Index \\
\hline I & $\begin{array}{l}\max R_{\mathrm{S}} \\
\text { s.t. } \mathrm{g}_{i}(x) \leq \mathrm{b}_{i}, \text { for } i=1, \ldots, m \\
\quad x \in \mathrm{X}\end{array}$ \\
\hline II & $\begin{array}{ll}\min & C_{\mathrm{S}} \\
\text { s.t. } & R_{\mathrm{S}} \geq R_{0} \\
& \mathrm{~g}_{i}(x) \leq \mathrm{b}_{i}, \text { for } i=1, \ldots, m \\
& x \in \mathrm{X}\end{array}$ \\
\hline III & $\begin{array}{l}\max \alpha R_{\mathrm{S}}+\beta C_{\mathrm{S}} \\
\text { s.t. } \mathrm{g}_{i}(x) \leq \mathrm{b}_{i}, \text { for } i=1, \ldots, m \\
\quad x \in \mathrm{X}\end{array}$ \\
\hline IV & $\begin{array}{l}\max \left[R_{\mathrm{S}}, C_{\mathrm{S}}\right] \\
\text { s.t. } \mathrm{g}_{i}(x) \leq \mathrm{b}_{i}, \text { for } i=1, \ldots, m \\
\quad x \in \mathrm{X}\end{array}$ \\
\hline \multicolumn{2}{|c|}{$\begin{array}{l}\mathrm{X}=\left(x_{1}, x_{2}, \ldots, x_{j}, \ldots, x_{n}\right) \\
x_{j}: \text { variable of subsystem } j \\
m: \text { number of resources } \\
n: \text { number of subsystems } \\
\mathrm{g}_{i}(x): \text { total amount of resource } i \text { required for } x \\
b_{i}: \text { resource target } i \\
R_{\mathrm{S}}: \text { system reliability, } R_{0}: \text { a specified target for } R_{\mathrm{S}} \\
C_{\mathrm{S}}: \text { selected resource, } C_{0}: \text { a specified minimum } C_{\mathrm{S}} \\
\alpha, \beta: \text { weighing coefficients }\end{array}$} \\
\hline
\end{tabular}

3) Type III: In this type, a linear combination of a system's reliability $R_{S}$ and selected resource cost $C_{S}$ is used as the objective function in a single-objective optimization problem. For the linear combination, coefficients $\alpha$ and $\beta$ are known as weighting parameters. With $\beta=0$, this formulation is equal to type I; when $\alpha=0$, this formulation is type II. For $\alpha>0$ and $\beta>0$, both reliability and the selected resource cost will be optimized, hence there could be three main possibilities: optimize $R_{S}$ and $C_{S}$ on an equal basis, intensify optimization of $R_{S}$ over $C_{S}$, or intensify optimization of $C_{S}$ over $R_{S}$. Therefore, weighting parameters can strengthen the optimization outcome towards reliability, the selected resource cost, or treat them with the same level of importance. The outcome of this type of formulation will vary according to the criteria selected.

4) Type $I V$ : This type corresponds to a multi-objective optimization problem. In the previous types, a reliability metric or cost was used as the objective function. On the contrary, multi-objective optimizes several metrics (reliability indexes or resources) simultaneously. Therefore, a mathematical expression in terms of several objectives is defined. Multiobjective optimization relies on finding a solution that satisfies constraints and optimize objectives that are usually in conflict 
with each other. For example, in the formulation $\max \left[R_{S}, C_{S}\right]$ of Table I, the objectives could be defined as: maximize system's reliability $R_{S}$, while system's weight resource $C_{S}$ is minimized. Minimizing resource usage while maximizing reliability usually brings to a trade-off between both because redundancy could improve reliability at the cost of heavier systems. There is extensive work in multi-objective optimization problem definition and solution search. This type of formulation does not have a unique structure, hence several sets of solutions known as Pareto front-sets are produced, which need further analysis to select the best trade-off [14].

Although type I and II have similar mathematical form, they define different kind of problems. The former aims to maximize reliability coupled to resource cost limitations, whereas the latter aims to minimize cost while ensuring a given reliability level. Type III is an option when the design needs to consider reliability and a selected resource cost at a higher level of importance in the optimization problem. The idea here is that the decision maker is willing to trade one off against the other, and knows this trade-off in advance (i.e. how much cost they are willing to pay to increase reliability by $\delta$. On the other hand, type IV states a multi-objective optimization problem that allows the user to choose between generated trade-off solutions once the potential solutions have been generated [12]. Type IV can be more useful if the acceptable trade-off cannot be easily determined beforehand, but cannot be automated to the same level - a decision is required from a decision maker at the end. From Table I, there are several possibilities for formulating a reliability-based optimization design problem. Reliability $R_{S}$ could be used in most cases at the design phase where the system is being constructed with a constrained amount of resources.

\section{DESIGN APPROACHES}

Reliability-based architecture design optimization has been applied in the design stage in several engineering fields. The main goal is to construct a system that could perform appropriately across all conditions and remain fault tolerant in order to complete its mission adequately with the least possible impact. Therefore, reliability plays a role of utmost importance in the design stage [11]. An aircraft power system is an autonomous microgrid containing turbine-driven generation, energy back-up and emergency systems, distribution network, power electronic converters, control and protection systems, and several types of loads, including loads which have a nonconstant profile, are non-linear, and are critical to safe aircraft operation [15]. The on-board electrical system must remain safe and reliable at all stages of the flight mission. Therefore, it is imperative to account for reliability in the architecture design optimization process, while still achieving the given resource cost targets (i.e. weight reduction, higher efficiencies, reducedsize components, etc.).

\section{A. Reliability optimization approach}

According to what was previously explained, reliability comprises the study of the overall system performance with failure-prone components. Substantial research effort has been concentrated in the attainment of bounds, approximations, and limit theorems for the reliability of a given system [16]. In fact, various optimal reliability design problems are known to be NPhard [17], [18]. Thus, heuristic approaches such as metaheuristics and genetic algorithms (GA) have been widely applied, along with work which combines GAs with heuristic algorithms, metaheuristics such as simulated annealing (SA), neural network techniques, and other local search methods. Despite the fact that exact approaches are not necessarily practical in reliability optimization problems, the development of exact methods, such as the branch-and-bound approach and lexicographic search, has recently concentrated on techniques to reduce the search space for the discrete optimization problem [17].

The reliability-based optimization design has not been applied in the aircraft power system architecture optimization literature. However, there have been profuse attempts to study this problem in microgrids. For instance, in [19] an unsatisfied demand hard-constraint index is used in a microgrid architecture optimization. Despite the fact that this formulation did not account for system contingencies (important in MEA power systems), it applied an energy-not-supplied reliability metric as a hard constraint, in a similar way as in type II of Table I. However, there are other reliability-oriented formulations that could be used as well. In the case of high voltage DC (HVDC) (one of the most promising concepts for MEA), parallel systems provide an effective solution for the development of a HVDC power system in MEA [20]. Additionally, as parallel systems have been thoroughly studied in reliability engineering, this concept can provide important insights in the design of MEA power systems, e.g. considering a load sharing capability. For example, in [21], a sufficient condition has been found to justify why a smaller number of components (withstanding a high load) is preferred over a larger number of components (withstanding a small load). Ideally, architecture optimization will explore connectivity schemes to comply with reliability and resource targets, but other approaches for pre-determined architectures are also possible. As an example, in [18] the optimal level of component reliability and the number of redundancies in a predefined architecture are determined.

There have been rigorous approaches for architecture optimization in the literature. Some contributions for improving current aircraft systems have been made based on the idea that industry adoption depends on the adaptability of new cuttingedge technologies to current systems. However, most of these proposals have been designed for AC power networks. In [22], 3-phase feeder reconfigurations and single-phase load rebalancing for weight-saving have been suggested and developed. Besides, there have been other attempts to improve MEA characteristics. Although MEA is a power system, the optimal power flow problem (OPF) cannot be assessed because generators and other energy sources are not competing to provide low cost power. In fact, there is no congestion in its electrical distribution system simply because there is no load growth or dispersity. Despite the fact that there have been some assessments on the performance of static and dynamic electrical models [23], [24], these formulations have not been directly related to the architecture optimization problem. Thus, power system-oriented formulations should be carefully considered in the optimal MEA architecture problem. 


\section{B. Reliability-based design formulation}

Although in the design stage of an aircraft power system it is desirable to optimize multiple objectives and comply with several constrained requirements, single-objective formulations could capture the essential features of a design problem. Reliability-based optimization methods considering $b i$-state systems and components could provide in-depth information about the characteristics of the design. On the other hand, if multi-state systems are considered, the problem would have a higher level of complexity that could only be approached with heuristics and approximation algorithms. In the case of systems with pre-determined architectures, several forms of reliability optimization problems could be used. For instance, a new arrangement of components to improve actual reliability performance can be found [25]. Indeed, this approach could be utilized in existing electrical networks where re-configuration of components could provide a rapid response assessment for control stability improvement during critical missions.

\section{MEA RELIABILITY-ORIENTED PROBLEM}

Nowadays, the European Union, in partnership with prominent industry leaders, has strongly been supporting the transition towards a more electrified aircraft product. Due to large scale research programs such as the Clean Sky 2 [2], more innovative technologies will unleash important benefits. Two of the most influential drivers in aircraft electrical architecture are the propulsion system [26]-[28], and the distribution system. Whilst some authors have highlighted the convenience of individual sub-system optimization, others have addressed the MEA optimization as a global problem (complete aircraft power system) [28]. Nevertheless, the fact is that less-efficient hydraulic and pneumatic power systems will be replaced by electrical counterparts in MEA in the near future [7]. Hence, the load increase in aircraft power systems will require reliable electrical networks that could perform at least at the same safety levels of today's aircrafts.

\section{A. Main formulatiosn for a MEA design example}

Several electrical configurations have been suggested since the concept of MEA emerged back in the 1950s [27]. A typical MEA power system could be sketched as in Fig. 1 from a system reliability perspective. This figure shows a parallel system where the power mainly flows from the source side (one or more generators) to the load side (one or more loads) through the distribution system. Then, it is possible to apply the formulations explained in section II for the system design, with a view to ensuring reliability as well as cost.

In Fig. 1, the source side is supplying the load side through several distribution components. Assuming that the source is $100 \%$ reliable (slack bus) and the load $P_{L O A D}$ is constant over the

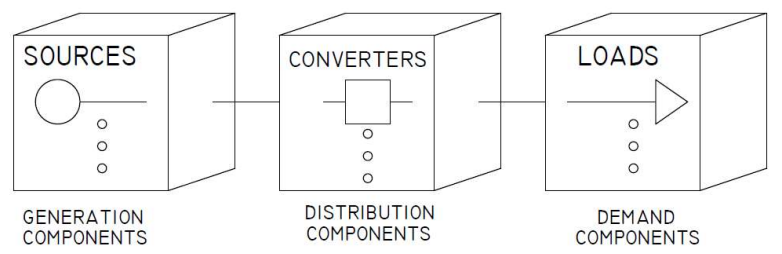

time period studied, this architecture can be translated into a single-objective optimization design problem.

Fig. 1. Reliability representation of a MEA power system.

Considering non-identical $N$ different and independent components in a parallel distribution system, the reliabilitybased architecture design optimization problem can be stated as in (1a)-(1f). There are three expressions that represent the constrained resources: weight $W(1 \mathrm{~b})$, efficiency $\eta(1 \mathrm{c})$, and power density $\rho[\mathrm{kW} / \mathrm{kg}](1 \mathrm{~d})$. In (1b), total system weight must be below a specified weight $W$; the efficiency of each chosen component must be at least $\Theta(1 \mathrm{c})$; the system power density should be at least $\rho$ (1d). The efficiency is a function of the power transferred through each component. The equality constraint (1e) is the power balance of the system, i.e. total load is supplied. Power transfer capacities for each component are ensured to be within limits in (1f). Formulation (1a) is similar to type I in Table I

The main purpose of this formulation is to maximize the probability that at least one component is working in the distribution system (1a). In other words, the chance of complete failure is reduced to a minimum. Given a set of different components with pre-determined weights $w_{i}$, power transfer capacity limits $\left[P_{i M A X}, P_{i M I N}\right]$, and reliability $R_{i}$, the optimization task is to maximize a system's reliability by choosing components to use and their corresponding powers. This is done through two vectors, $X$ and $P$. Vector $S=\left[s_{1}, s_{2} \ldots s_{N}\right]$ contains the assignment of $\{0,1\}$ values to the variables $s_{i}$, where 0 is used if the $\mathrm{i}^{\text {th }}$ component is not chosen and 1 is used if it is chosen. Vector $P=\left[P_{1}, P_{2} \ldots P_{N}\right]$ contains the assignment of a power value $P_{i}$ within $\left[P_{i M A X}, P_{i M I N}\right]$ for each $i^{\text {th }}$ component. Therefore, the vector $V=[S, P]$ represents a solution vector for this problem (given $N$ components).

$$
\begin{aligned}
& \max _{V}\left[1-\prod_{i=1}^{N}\left(1-R_{i} s_{i}\right)\right] \\
& \text { s.t. } \sum_{i=1}^{N} w_{i} s_{i} \leq W \\
& \\
& \eta_{i}\left(P_{i}\right) \geq \Theta s_{i} \quad \text { for } i=1,2, \ldots, N \\
& \frac{P_{L O A D}}{\sum_{i=1}^{N} w_{i} s_{i}} \geq \rho \\
& \sum_{i=1}^{N} P_{i} s_{i}=P_{L O A D} \\
& P_{i \min } \leq P_{i} \leq P_{i \max }
\end{aligned}
$$

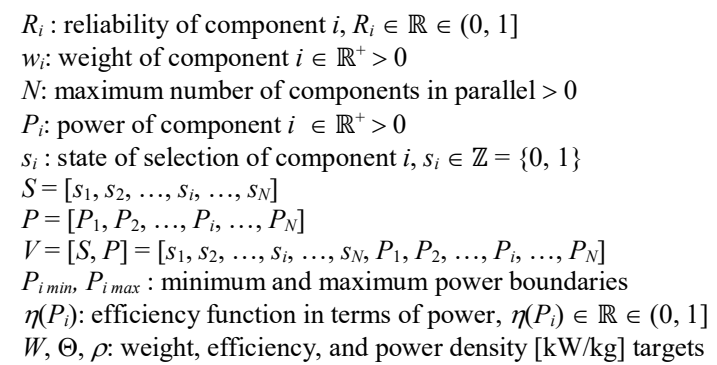

The solution to this formulation is the vector $V=[S, P]$, where $S$ is the selection vector and $P$ is the power vector, such 
that all the constraints are met and the system's reliability is maximized. As an example, if a 4-component set with the characteristics of (2a)-(2c), and the constrained targets (3) are considered, the optimal solution for the reliability-based optimization design power system architecture problem in (1a) that comply with all the constraints (3) is shown in (4).

$$
\begin{aligned}
& R=[0.91, \quad 0.85, \quad 0.90,0.87] \\
& w=[1.554,1.337,1.579,1.456] \\
& \eta_{1}=0.8566+0.2400 P_{1}-0.1145 P_{1}^{2} ; 0.5<P_{1}<1.5 \\
& \eta_{2}=0.7710+0.4190 P_{2}-0.2045 P_{2}^{2} ; 0.6<P_{2}<1.6 \\
& \eta_{3}=0.6040+0.8387 P_{3}-0.4674 P_{3}^{2} ; 0.4<P_{3}<1.4 \\
& \eta_{4}=0.5044+1.1626 P_{4}-0.6715 P_{4}^{2} ; \quad 0.6<P_{4}<1.6 \\
& W=3.25 \mathrm{~kg} ; \quad \Theta=0.92 ; \quad \rho=0.95 \frac{\mathrm{kW}}{\mathrm{kg}} ; \quad P_{L O A D}=2.9 \mathrm{~kW} \\
& S=[1,1,0,0] \quad P=[1.4,1.5,0,0] \mathrm{kW} \\
& \text { optimal } \boldsymbol{R}_{\text {system }}=0.9865 \text {, with } \boldsymbol{w}=2.8910 \mathrm{~kg}
\end{aligned}
$$

If one changes the formulation in (1a), and uses instead the weight resource cost as the optimization objective, the problem could be stated as in (5a):

$$
\begin{array}{ll}
\min _{V}\left[\sum_{i=1}^{N} w_{i} s_{i}\right] \\
\text { s.t. } 1-\prod_{i=1}^{N}\left(1-R_{i} s_{i}\right) \geq R_{0} \\
& \eta_{i}\left(P_{i}\right) \geq \Theta s_{i} \quad \text { for } i=1,2, \ldots, N \\
& \frac{P_{L O A D}}{\sum_{i=1}^{N} w_{i} s_{i}} \geq \rho \\
& \sum_{i=1}^{N} P_{i} s_{i}=P_{L O A D} \\
& P_{i \min } \leq P_{i} \leq P_{i \text { max }}
\end{array}
$$

In the above formulation (5a) the weight of the system is minimized, in a similar way to the type II in Table I (weight is the selected constrained resource). Now the system's reliability is written as a hard-constraint (5b), and it must be at least $R_{0}$. The rest of the constraints remain with the same meaning as before. If the component set of (2a)-(2c) is utilized, the solution for the reliability-based optimization design power system architecture problem in (5a) that complies with the constrained targets (6) is shown in (7).

$$
\begin{aligned}
& R_{0}=0.9870 ; \quad \Theta=0.92 ; \quad \rho=0.70 \frac{\mathrm{kW}}{\mathrm{kg}} ; \quad P_{L O A D}=2.5 \mathrm{~kW} \\
& S=[1,0,1,0] \quad P=[1.3,0,1.2,0] \mathrm{kW} \\
& \text { optimal } \boldsymbol{w}=3.133 \mathrm{~kg} \text {, with } \boldsymbol{R}_{\text {system }}=\mathbf{0 . 9 9 1 0}
\end{aligned}
$$

The examples above show how the theory detailed in previous sections can be used to derive formulations which can be utilized to design reliability-oriented power system architectures for MEA. Furthermore, other kinds of solutions can be obtained if linear combination of objectives (type III, Table I) and multi-objective (type IV, Table I) optimization are used.

\section{B. Future considerations}

Formulations (1a) and (5a) propose that if there is at least one component available, the system will succeed. Whilst this is true in highly redundant systems, it is not totally valid in aircraft networks. The formulations (1a) and (5a) could be modified to handle a load-sharing capability to enhance the MEA power system characteristic. Such a load-sharing capability could help in improving the size of the components since the load could be supplied by a group of smaller components. The cost of including this load-sharing feature in (1a) and (5a) is that the reliability $R_{i}$ is no longer constant, but changes in time due to the fact that reliability of components under higher load-levels is different. This formulation could provide an improvement to proposals such as More Open Electric Technologies (MOET) MEA electrical system [29].

In addition, other convenient approaches for the system in Fig. 1 also need investigating. For instance, it can be approximated to a weighted system (or a variation of a weighted system) [30]. This representation is more complex than the system's reliability of (1a) and (5b). However, one of the advantages is that the weight could represent the power being transferred in each component. Given the fact that the electrical distribution grid performs power conversion and energy transportation from sources to loads [7], a weighted system is a closer representation of power and energy flows. Moreover, it could handle electrical parameters conveniently, i.e. generator load, converter flow [5], and demand consumption. If other novel technologies were included (i.e. storage devices, fuel cells, and supercapacitors), bi-directional power flows could also be included in weighted systems to increase system energy efficiency and overall system reliability [26]. Therefore, there is still a great opportunity to explore reliability-based formulations in the MEA power system optimization problem and these will be reported in future papers.

\section{CONCLUSIONS}

There is a huge potential for reliability-based design optimization problems in MEA power systems. This paper has reviewed four main reliability optimization formulations used in the design of engineering systems and outlined their potential for use in aircraft power system architecture optimization. An electrical power system architecture design problem for MEA has been successfully stated as a reliability-based optimization problem including technical constraints. This formulation has been analyzed and two design cases have been presented for a given set of four components. The optimization outcome has provided solutions to design a MEA power system. Further investigations for the optimization of more realistic power systems with enhanced capabilities and risk assessments will be performed in the future.

\section{ACKNOWLEDGMENT}

This work is funded by the INNOVATIVE doctoral programme. The INNOVATIVE programme is partially funded by the Marie Curie Initial Training Networks (ITN) action, and partially by the Institute for Aerospace Technology (IAT) at the University of Nottingham. 


\section{REFERENCES}

[1] X. Roboam, B. Sareni, and A. De Andrade, "More Electricity in the Air," Industrial Electronics Magazine, IEEE, vol. 6, no. 4, pp. 6-17, Dec-2012.

[2] Clean Sky, "Clean Sky," European Union Funding for Research and Innovation, 2018. [Online]. Available: http://www.cleansky.eu. [Accessed: 10-May-2018].

[3] Airbus, "A380 - Benefits to Airlines,” 2018. [Online]. Available: http://www.airbus.com/aircraft/passenger-aircraft/a380family/benefits-to-airlines.html.

[4] C. Soutis, "Fibre reinforced composites in aircraft construction," Prog. Aerosp. Sci., vol. 41, no. 2, pp. 143-151, 2005.

[5] X. Zhao, J. M. Guerrero, and X. Wu, "Review of aircraft electric power systems and architectures," in 2014 IEEE International Energy Conference (ENERGYCON), 2014, pp. 949-953.

[6] P. Wheeler and S. Bozhko, "The more electric aircraft: Technology and challenges," IEEE Electrification Magazine, vol. 2, no. 4, pp. 6-12, Dec-2014.

[7] M. Tooley and D. Wyatt, Aircraft Electrical and Electronic Systems- Principles, maintenance and operation, 1 st ed. Oxford, UK: Elsevier Ltd., 2009.

[8] L. Laguna, R. Prieto, J. A. Oliver, J. A. Cobos, H. Visairo, and P. Kumar, "Power Conversion Modeling Methodology Based on Building Block Models," in Energy Conversion Congress and Exposition (ECCE), 2009 IEEE, 2009, pp. 3404-3410.

[9] I. Moir and A. Seabridge, Design and Development of Aircraft Systems, 2nd ed. New Jersey: Wiley, 2012.

[10] A. Engel, Y. Reich, T. R. Browning, and D. M. Schmidt, "Optimizing system architecture for adaptability," in Proceedings of International Design Conference, DESIGN, 2012, vol. DS 70, pp. $1677-1688$.

[11] W. Kuo, V. R. Prasad, F. A. Tillman, and C. L. Hwang, Optimal Reliability Design- Fundamentals and Applications, 1st ed. Cambridge University Press, 2001.

[12] B. W. Kuo and R. Wan, "Optimal Reliability Design-Modeling," in Encyclopedia of Statistics in Quality and Reliability, 1st ed., Knoxville: John Wiley \& Sons, 2008, pp. 1-13.

[13] K. O. Kim and W. Kuo, "Percentile life and reliability as performance measures in optimal system design," IIE Trans. (Institute Ind. Eng., vol. 35, no. 12, pp. 1133-1142, 2003.

[14] G. Chiandussi, M. Codegone, S. Ferrero, and F. E. Varesio, "Comparison of multi-objective optimization methodologies for engineering applications," Comput. Math. with Appl., vol. 63, no. 5, pp. 912-942, 2012.

[15] I. Moir and A. Seabridge, Aircraft Systems- Mechanical, electrical, and avionics subsystems integration, 3rd ed. West Sussex, UK: John Wiley \& Sons, 2008

[16] M. V Boutsikas and M. V Koutras, "Reliability Approximation for
Markov Chain Imbeddable Systems," Methodol. Comput. Appl. Probab., vol. 2, no. 4, pp. 393-411, 2000 .

[17] B. W. Kuo and R. Wan, "Optimal Reliability Design-Algorithms and Comparisons," in Encyclopedia of Statistics in Quality and Reliability, 1st ed., Knoxville: John Wiley \& Sons, 2008, pp. 1-11.

[18] F. A. Tillman, C. L. Hwang, and W. Kuo, "Determining Component Reliability and Redundancy for Optimum System Reliability," IEEE Trans. Reliab., vol. R-26, no. 3, pp. 162-165, 1977.

[19] J. Mitra, S. B. Patra, and S. J. Ranade, "Reliability Stipulated Microgrid Architecture Using Particle Swarm Optimization," in 2006 International Conference on Probabilistic Methods Applied to Power Systems, 2006, pp. 1-7.

[20] F. Gao and S. Bozhko, "Modeling and Impedance Analysis of a Single DC Bus-Based Multiple-Source Multiple-Load Electrical Power System,” IEEE Trans. Transp. Electrif., vol. 2, no. 3, 2016.

[21] K. O. Kim, "Optimal number of components in a load-sharing system for maximizing reliability," J. Korean Stat. Soc., vol. 47, no. 1, pp. 32-40, 2018.

[22] M. Terorde, A. Lucken, and D. Schulz, "Weight saving in the electrical distribution systems of aircraft using innovative concepts," Int. J. energy Res., vol. 31, no. August 2007, pp. 135 $147,2007$.

[23] K. L. Xu, N. Xie, C. M. Wang, J. W. Deng, and X. D. Shi, "Static modeling and power flow of the more electric aircraft power system," in $20163 \mathrm{rd}$ International Conference on Systems and Informatics, ICSAI 2016, 2017, no. 1, pp. 193-198.

[24] Z. Yang, J. Qu, Y. Ma, and X. Shi, "Modeling and Simulation of power distribution system in More Electric Aircraft," J. Electr. Comput. Eng., vol. 2015, no. 1, pp. 1-7, 2015.

[25] R. Medjoudi, H. Bediaf, and D. Aissani, "Power System Reliability: Mathematical Models and Applications," in Power System Reliability: Mathematical Models and Applications, 1st ed., C. Volosencu, Ed. IntechOpen, 2017.

[26] B. Sarlioglu and C. T. Morris, "More Electric Aircraft: Review, Challenges, and Opportunities for Commercial Transport Aircraft," IEEE Trans. Transp. Electrif., vol. 1, no. 1, pp. 54-64, 2015.

[27] V. Madonna, P. Giangrande, and M. Galea, "Electrical Power Generation in Aircraft : review , challenges and opportunities," IEEE Trans. Transp. Electrif., 2018.

[28] A. S. Gohardani, G. Doulgeris, and R. Singh, "Challenges of future aircraft propulsion: A review of distributed propulsion technology and its potential application for the all electric commercial aircraft," Prog. Aerosp. Sci., vol. 47, no. 5, pp. 369-391, 2011.

[29] T. Jomier, "More Open Electrical Technologies," 2009.

[30] W. Kuo and M. J. Zuo, Optimal Reliability Modeling: Principles and Applications, 1st ed. Hoboken, New Jersey: John Wiley \& Sons, 2003. 